\title{
Carbapenemase-Producing Enterobacteriaceae
}

\author{
Yohei Doi, MD, $\mathrm{PhD}^{1}$ David L. Paterson, MBBS, PhD, FRACP, FRCPA ${ }^{2,3}$ \\ ${ }^{1}$ Division of Infectious Diseases, University of Pittsburgh School of \\ Medicine, Pittsburgh, Pennsylvania \\ 2 The University of Queensland Centre for Clinical Research, Royal \\ Brisbane and Women's Hospital, Brisbane, Australia \\ Address for correspondence Yohei Doi, MD, PhD, Division of \\ Infectious Diseases, Department of Medicine, University of Pittsburgh \\ School of Medicine, S829 Scaife Hall, 3550 Terrace Street, Pittsburgh, \\ PA 15261 (e-mail: yod4@pitt.edu).
}

${ }^{3}$ Centre for Healthcare Related Infection Surveillance and Prevention (CHRISP), Brisbane, Australia

Semin Respir Crit Care Med 2015;36:74-84.

\begin{abstract}
Keywords

- antimicrobial resistance

- carbapenemases

- Klebsiella pneumoniae

- Enterobacteriaceae

Carbapenemase-producing Enterobacteriaceae (CPE) were almost nonexistent up to the 1990s, but are today encountered routinely in hospitals and other healthcare facilities in many countries including the United States. KPC-producing Klebsiella pneumoniae was the first to emerge and spread globally and is endemic in the United States, Israel, Greece, and Italy. Recently, NDM-producing Enterobacteriaceae and OXA-48-producing K. pneumoniae appear to be disseminating from South Asia and Northern Africa, respectively. They are almost always resistant to all $\beta$-lactams including carbapenems and many other classes. Mortality from invasive CPE infections reaches up to $40 \%$. To obtain the maximal benefit from the limited options available, dosing of antimicrobial agents should be optimized based on pharmacokinetic data, especially for colistin and carbapenems. In addition, multiple observational studies have associated combination antimicrobial therapy with lower mortality compared with monotherapy for these infections. The outcomes appear to be especially favorable when patients are treated with a carbapenem and a second agent such as colistin, tigecycline, and gentamicin, but the best approach is yet to be defined.
\end{abstract}

Carbapenem resistance in Enterobacteriaceae had been a negligible phenomenon before 2000. Back then, the rare occurrence of reduced susceptibility to carbapenems in Enterobacteriaceae was mostly attributed to a combination of production of extended-spectrum $\beta$-lactamase or AmpC $\beta$ lactamase and deficiency of porins in the outer membrane. ${ }^{1,2}$ Reports on carbapenem resistance due to production of carbapenemases ( $\beta$-lactamases capable of hydrolyzing carbapenems), such as IMP or VIM-type metallo- $\beta$-lactamases, were beginning to emerge, ${ }^{1}$ but the prevalence of carbapenemase-producing Enterobacteriaceae (CPE) was exceedingly low. This picture changed when Klebsiella pneumoniae producing KPC-type carbapenemase appeared in the late 1990s and spread worldwide in the 2000s, ${ }^{3}$ recently followed by expansion of Enterobacteriaceae producing NDM-type carbapenemase and K. pneumoniae producing OXA-48-type carbapenemase. These organisms are almost always resistant to carbapenems and many other classes of commonly used antimicrobial agents; thus, managing infections caused by them poses a substantial challenge in clinical practice. In this review, we will briefly examine the epidemiology and microbiology of these emerging CPEs, and review the current knowledge regarding their clinical management, including prevention and treatment.

\section{Epidemiology of Carbapenemase-Producing Enterobacteriaceae}

\section{KPC-Producing $K$. pneumoniae}

KPC stands for Klebsiella pneumoniae carbapenemase and is a class A $\beta$-lactamase which has the capacity to hydrolyze penicillins, cephalosporins, and carbapenems. KPC was initially reported from a K. pneumoniae strain isolated in North Carolina in $1996 .^{3}$ By 1997, KPC-producing K. pneumoniae
Issue Theme Antimicrobial Resistance: Management of Superbugs; Guest Editor, David L. Paterson, MBBS, PhD, FRACP, FRCPA
Copyright @ 2015 by Thieme Medical Publishers, Inc., 333 Seventh Avenue, New York, NY 10001, USA. Tel: +1(212) 584-4662.
DOI http://dx.doi.org/ 10.1055/s-0035-1544208. ISSN 1069-3424. 
appeared in some hospitals in New York City, ${ }^{4}$ and continued to spread. ${ }^{5}$ In a city-wide surveillance study conducted in 2006 , 38\% of K. pneumoniae clinical isolates produced KPC, making this a truly concerning epidemic. ${ }^{5}$ KPC-producing $K$. pneumoniae has since spread across the United States. ${ }^{6}$ but not evenly, with eastern census regions showing higher prevalence than western and southern regions. KPC-producing K. pneumoniae appears to be especially endemic in the mid-Atlantic, Midwest regions, as well as in Florida and Puerto Rico. The overall prevalence of carbapenem resistance among Klebsiella spp. isolates causing hospital-acquired infections in U.S. hospitals was approximately $12 \%$ between 2009 and 2010 according to data from the National Healthcare Safety Network. ${ }^{7}$

KPC-producing K. pneumoniae has since spread worldwide. The first country besides the United States that experienced a nationwide outbreak was Israel. ${ }^{8}$ Emergence and a sharp increase in the number of KPC-producing K. pneumoniae isolates was identified in Tel Aviv hospitals between 2005 and 2006, which were shown to be genetically related to the isolates circulating in U.S. hospitals. ${ }^{9}$ It was apparent by early 2007 that this was a nationwide outbreak, which led the Ministry of Health to issue infection control guidelines mandating contact precaution of hospitalized carriers and the use of dedicated staffing which was enforced by a task force. ${ }^{10}$ These measures were successful in controlling the nationwide incidence of KPC-producing K. pneumoniae acquisitions, and the downward trend has continued as of $2012 .^{11}$

The other countries heavily affected by this organism include Greece and Italy. In Greece, a hospital outbreak was detected in 2007, ${ }^{12}$ and a nationwide epidemic ensued. Carbapenem resistance rate of 60.5\% was recorded in 2012 for $K$. pneumoniae in Greece in the ERAS-Net surveillance (http://www.ecdc.europa.eu). While KPC-producing K. pneumoniae accounts for the majority of this, K. pneumoniae producing VIM-type metallo- $\beta$-lactamase is also endemic in Greece, ${ }^{13}$ further complicating the picture. Italy also now has a high rate of carbapenem resistance among K. pneumoniae (28.8\% in the above surveillance report), following initial reports of hospital outbreak occurring around 2009. ${ }^{14}$ Nearly $90 \%$ of them are producing KPC. ${ }^{15}$ High burdens of KPCproducing K. pneumoniae have also reported from China, Brazil, and Colombia. ${ }^{16}$

The rapid global spread of KPC-producing K. pneumoniae is now understood as a largely clonal phenomenon. ${ }^{17} \mathrm{~A}$ specific clone of KPC-producing K. pneumoniae, called ST258, is globally distributed. ST stands for sequence type, and is assigned by multilocus sequence typing, which is a nucleotide sequence-based bacterial typing method where seven genes on the chromosome are sequenced to decipher global relatedness among strains. ST258 predominates among KPC-producing K. pneumoniae in the United States. ${ }^{6}$ ST258 as well as ST512, which is closely related to ST258, has been found commonly in Israel and Italy, ${ }^{18,19}$ whereas ST11 and ST437 appear to predominate in China and Brazil, respectively. ${ }^{20,21}$ These STs are all closely related to ST258 suggesting the presence of a common origin, most likely in the mid-Atlantic United States. On the other hand, plasmids carrying the KPC gene are diverse in structure and often capable of selftransmission to other strains by conjugation. ${ }^{22}$

While carbapenem resistance mediated by KPC production is most conspicuous in K. pneumoniae, the KPC gene can be acquired by other species of Enterobacteriaceae including Enterobacter spp. and Escherichia coli, ${ }^{23,24}$ and on rare occasions Pseudomonas aeruginosa and Acinetobacter baumannii as well. ${ }^{25}$ Among these species, the potential of $E$. coli acquiring KPC is especially concerning because of its community-wide distribution as a commensal organism.

\section{NDM-Producing Enterobacteriaceae}

NDM stands for New Delhi metallo- $\beta$-lactamase. It is a class B $\beta$-lactamase and is capable of hydrolyzing penicillins, cephalosporins, and carbapenems, but unlike KPC, it does not hydrolyze aztreonam. NDM-producing K. pneumoniae and E. coli were first identified in an Indian patient residing in Sweden who had hospitalization for wound infections in New Delhi before returning to Sweden in early $2008 .^{26}$ It was soon reported that NDM-producing Enterobacteriaceae were present in Indian hospitals as early as $2006 .{ }^{27}$ In a study where Enterobacteriaceae clinical isolates were collected from three Indian hospitals in 2010, the prevalence of the NDM production was $5.2 \%{ }^{28}$ In Pakistan, prevalence of $18.5 \%$ has been reported for NDM-producing Enterobacteriaceae among stool samples collected at two hospitals in $2010 .^{29}$ In addition to the Indian Subcontinent as the primary reservoir, it has been speculated that secondary but significant reservoirs of NDMproducing organisms might exist in the Balkans and the Gulf region. ${ }^{30,31}$ NDM-producing bacteria have now been reported from all corners of the world, including the United States, therefore having shown even more rapid spread compared with those producing KPC since the initial appearance. NDM is also distinct from KPC in that its spread is occurring both in healthcare settings and the community. NDM-producing organisms have been detected in tap water and seepage samples collected in New Delhi, ${ }^{32}$ and in species that are considered community-acquired pathogens, such as Salmonella enterica and Vibrio cholerae. ${ }^{33,34}$

\section{OXA-48-Producing $K$. pneumoniae}

OXA stands for oxacillinase and is a diverse group of $\beta$ lactamases classified to class D. Some of OXA $\beta$-lactamases additionally have the capability to hydrolyze carbapenems. In Enterobacteriaceae, OXA-48 and its related enzymes are the ones that require attention. OXA-48 was first found in a $K$. pneumoniae strain isolated in Turkey in $2001 .{ }^{35}$ Its production mediates resistance to penicillins and carbapenems (especially imipenem), but not to cephalosporins. The OXA-48 gene is usually encoded on a plasmid and may spread to other species of Enterobacteriaceae, but most cases have been reported in K. pneumoniae. OXA-48-producing K. pneumoniae often also coproduces an extended-spectrum $\beta$-lactamase. These isolates are then resistant to all $\beta$-lactams including cephalosporins. K. pneumoniae producing OXA-48 and its related $\beta$-lactamases have been reported mostly from Turkey, North Africa, and recently the Gulf region and India. ${ }^{30,36}$ Outbreaks have occurred in Europe as well. However, their 
prevalence has not been estimated, mostly because of the difficulty in detecting OXA-48-producing isolates, which could present with variable levels of carbapenem resistance and may remain susceptible to cephalosporins. ${ }^{36}$ They remain extremely rare in the United States, but imported cases have been reported. ${ }^{37}$

\section{Detection of Carbapenemase-Producing Enterobacteriaceae}

CPE may or may not be frankly "resistant" to carbapenems. This is because, while production of carbapenemase always elevates the minimum inhibitory concentrations (MICs) of carbapenems, they may not be high enough to be called resistant or intermediately resistant. To address this issue, the Clinical and Laboratory Standards Institute (CLSI) lowered the MIC breakpoints for carbapenems in 2010. For example, an MIC of $16 \mathrm{mg} / \mathrm{L}$ was required for meropenem resistance prior to 2010, whereas an MIC of $4 \mathrm{mg} / \mathrm{L}$ has been defined as resistant since this revision. However, the uptake of the revised breakpoints has been slow, mostly because of validation delays on commercially available, automated susceptibility testing instrument. ${ }^{38}$ It has been well documented that a substantial portion of CPE is missed by the old breakpoints. ${ }^{39}$ In general, resistance to ertapenem (under the current breakpoints) is considered to have the best sensitivity but less than ideal specificity in screening of carbapenemaseproducing isolates. ${ }^{39}$ Therefore, those that are resistant or intermediately resistant (i.e., MIC of $\geq 1 \mathrm{mg} / \mathrm{L}$ ) should then undergo confirmatory testing for carbapenemase production.

In Europe, the European Committee on Antimicrobial Susceptibility Testing (EUCAST) defines susceptibility to imipenem and meropenem as an MIC $\leq 2 \mathrm{mg} / \mathrm{L}$, with resistance being defined as an MIC $>8 \mathrm{mg} / \mathrm{L}$. The EUCAST guidelines note that in many areas, carbapenemase detection and characterization is recommended or mandatory for infection control purposes. ${ }^{39}$

There are several approaches to confirmatory testing, using either molecular or nonmolecular methods. In clinical microbiology laboratories, nonmolecular methods are more feasible because they do not require expensive instrument and reagents. The most widely adopted and endorsed by the CLSI is the modified Hodge test. ${ }^{40}$ It is a culture-based test to detect release of carbapenemase into agar media and can be performed without any special equipment or reagent, but the interpretation of the results may be subjective. The test performs reasonably well with KPC-producing K. pneumoniae, but false-positive results are frequent in Enterobacter spp. One caveat is that this test is designed to detect carbapenemase in general and not specifically KPC. In countries with high burden of KPC producers, a positive test is likely to result from KPC production, but could on rare occasions result from production of OXA-48, NDM, or other infrequent metallo- $\beta$ lactamases. A positive test should therefore be reported out as "carbapenemase producer" and not "KPC producer."

A rapid chromogenic test ("Carba NP test") has also been developed, where hydrolysis of imipenem by crude carbapenemase extracted from the isolates is observed by color changes in a microtiter well. ${ }^{41}$ This test has equivalent sensitivity and superior specificity in comparison with the modified Hodge test, ${ }^{42}$ and has the added advantage of not needing an extra day of culturing to read the results.

In countries or regions where KPC-producing organisms are endemic, inhibitor-based testing using a boronic acid compound is a viable alternative to the aforementioned methods. Boronic acid was initially studied as an inhibitor of AmpC $\beta$-lactamases, but was later found to also inhibit the activity of KPC. Therefore, by adding aminophenyl boronic acid $(300$ or $400 \mu \mathrm{g}$ ) to a carbapenem disk (ertapenem or meropenem), enlargement of the inhibitory zone in comparison with a carbapenem disk alone can be used to infer KPC activity. ${ }^{43,44}$ In this case, a positive test can be reasonably signed out as "KPC producer." This test would not detect NDM or OXA-48-producing isolates.

Phenotypic detection of NDM, which is a metallo- $\beta$-lactamases, depends on the use of a zinc chelator such as ethylenediaminetetraacetic acid (EDTA), sodium mercaptoacetic acid (SMA), and dipicolinic acid, where these compounds are used to inhibit the activity of NDM. ${ }^{45}$ Etest MBL strips, which use EDTA as the inhibitor, are commercially available. Currently, no nonmolecular test for detection of OXA-48 is available.

Definitive identification of carbapenemases in Enterobacteriaceae still relies on nucleic acid-based tests, including PCR. PCR-based commercial test kits are now available (e.g., CheckPoints products). ${ }^{46}$

\section{Active Screening of CPE Carriage}

While not routinely performed in most hospitals, active screening of CPE carriage may need to be considered under circumstances where there is a high incidence rate of CPE infection and ongoing transmission within the hospital. Early detection of CPE carriage allows for implementation of appropriate infection control measures in a timely manner before transmission occurs. Patients who are considered to be at high risk for acquiring CPE include contacts of newly discovered carriers, those transferred from another hospital with high incidence of $\mathrm{CPE}$ infection, and those hospitalized in ICUs or floors (wards) with high incidence of CPE infection. Rectal swab or stool is the preferred specimen for surveillance because Enterobacteriaceae constitute the intestinal microbiota. They are typically plated on selective medium that preferentially grows CPE. Examples include direct inoculation of a MacConkey plate with an ertapenem disk on it, ${ }^{47}$ direct inoculation of a MacConkey plate containing $1 \mathrm{mg} / \mathrm{L}$ of imipenem, ${ }^{48}$ and overnight enrichment in broth containing an imipenem disk followed by plating on a MacConkey plate. ${ }^{49}$ Commercially manufactured selective media include CHROMagar KPC (CHROMagar, Paris, France) and Spectra CRE (Oxoid, Basingstoke, Hampshire, UK), where these specimens can be directly cultured on these plates. While these chromogenic media allow for distinction between K. pneumoniae, E. coli, and lactose-non-fermenters, they do not identify species or type of carbapenemase. PCR-based detection of the KPC and NDM genes has also been proposed and may be 
more sensitive than culture-based methods, ${ }^{50}$ but the higher costs would suggest that they may be appropriate in settings with high prevalence of CPE.

\section{Antimicrobial Agents Used for Treatment of CPE Infections}

\section{General Considerations}

Carbapenems are often reserved for treatment of infections caused by otherwise drug-resistant organisms as efficacious and safe agents of last resort. Carbapenem resistance due to production of carbapenemase thus leaves clinicians and patients with very few treatment options. With few exceptions, these carbapenemases render penicillins (including $\beta$ lactamase combinations such as piperacillin-tazobactam) and cephalosporins (including cefepime) inactive, effectively wiping out the entire $\beta$-lactams from therapeutic consideration, at least in the context of standalone therapy. In terms of in vitro susceptibility, two agents that maintain activity relatively well across CPEs are polymyxins (colistin and polymyxin B) and tigecycline. Fosfomycin and doxycycline (or minocycline) are active against some CPE strains and their use could be considered especially in the case of urinary tract infection. In addition, some, but not all, KPC-producing $K$. pneumoniae strains remain exquisitely susceptible to gentamicin. However, this is not the case for NDM-producing Enterobacteriaceae strains, many of which produce $16 \mathrm{~S}$ ribosomal RNA methyltransferase that render them highly resistant to all aminoglycosides including gentamicin. Finally, although OXA-48 itself does not hydrolyze cephalosporins well, OXA-48-producing $K$. pneumoniae strains appear to coproduce ESBL in most instances, and as a result are resistant to cephalosporins as well as carbapenems.

\section{Polymyxins (Colistin and Polymyxin B)}

Polymyxins are cationic cyclic polypeptides linked to a fatty acid chain. Colistin and polymyxin B differ only by one amino acid and share similar biological activity, and exert their bactericidal activity by binding to lipid A of lipopolysaccharide followed by uptake across the outer membrane. ${ }^{51}$ They were discovered over 50 years ago and were used for treatment of gram-negative bacterial infection. The advent of safer alternatives (e.g., cephalosporins) since led to their disuse, but they have been brought back to widespread use since the turn of the century for treatment of infection caused by carbapenemresistant bacteria including CPE as well as $P$. aeruginosa and $A$. baumannii. Colistin is active against most gram-negative bacterial species, except for Proteus spp., Providencia spp., and Serratia spp., which are intrinsically resistant. Both colistin and polymyxin $B$ are available in clinical use in some countries in the Americas including the United States, but only colistin is available in many countries worldwide. Therefore, more data are available on colistin compared with polymyxin B. Major adverse effects are nephrotoxicity and neurotoxicity. Nephrotoxicity occurs in as many as $50 \%$ of patients and more among critically ill patients, ${ }^{52,53}$ but these rates may not be higher than controls overall. ${ }^{54}$ It is usually reversible upon discontinuation of the agents. Neurotoxicity such as neuromuscular blockade may also occur with polymyxin use, but they appear to be rare events in recent clinical series. ${ }^{54}$

Polymyxin B is administered to patients as the active drug, but colistin is administered as its prodrug colistin methanesulfonate (CMS), which sequentially undergoes hydrolysis to form a mixture of partially sulfomethylated as well as active colistin. The complex pharmacokinetics of CMS and colistin began to be elucidated only recently and remain a subject of intensive investigation. The half-life of CMS and colistin is approximately 2.2 and 18.5 hours, respectively. ${ }^{55} \mathrm{CMS}$ undergoes net tubular secretion with extensive renal clearance, whereas colistin is subjected to tubular reabsorption and its clearance is largely nonrenal. Only approximately $7 \%$ of the administered CMS is converted to colistin systemically. ${ }^{56}$ As a result, the maximum concentration of colistin in plasma after the first dose given at $90 \mathrm{mg}$ colistin base activity (CBA) is only $0.6 \mathrm{mg} / \mathrm{L}$, well below the MICs of many organisms that require therapy with this agent, and colistin is undetectable in bronchoalveolar lavage 2 hours after administration of CMS. ${ }^{57,58}$ When CMS is given at 90 mg CBA every 8 hours, the steady state concentration of approximately $2.3 \mathrm{mg} / \mathrm{L}$ is achieved only after 2 or 3 days of therapy. If the first dose is given at $180 \mathrm{mg}$ CBA instead of $90 \mathrm{mg}$ as a loading dose, then the maximum colistin concentration of approximately 1.3 $\mathrm{mg} / \mathrm{L}$ is achieved in 8 hours. ${ }^{55}$ It therefore appears that a loading dose is warranted to obtain a therapeutic plasma concentration of colistin in a timely manner. Another consideration is that a combination with another active agent is likely to be beneficial in improving patient outcome and preventing development of resistance, unless colistin MIC is very low. In addition, colistin does not achieve therapeutic levels in the cerebrospinal fluid via intravenous administration. Treatment of meningitis thus requires either intrathecal or intraventricular routes of administration.

Much less is known about the pharmacokinetics of polymyxin $\mathrm{B}$, which is administered as the active drug upfront. It has a half-life of approximately 13.6 hours and is cleared mostly via nonrenal routes. ${ }^{59}$

Most CPE isolates remain susceptible to polymyxins, but colistin-resistant KPC-producing K. pneumoniae strains are increasingly reported. ${ }^{60-62}$ Colistin-resistant K. pneumoniae producing NDM or OXA-48 appear to be rarer but have been reported as well. ${ }^{63,64}$ These cases are mostly reported in those who have been exposed to colistin. Therefore, it would be prudent to test colistin susceptibility if the patient has been treated with colistin or polymyxin B before.

\section{Tigecycline}

Tigecycline is a derivative of minocycline designed to circumvent efflux-mediated resistance mechanisms. It has a broad spectrum of activity against gram-positive and gram-negative bacteria, including CPE. However, nonsusceptibility to tigecycline is increasingly common in KPC-producing $K$. pneumoniae, ${ }^{65}$ occurring in patients who have been treated with this agent. ${ }^{66}$

Tigecycline has a large volume of distribution resulting in low concentrations in blood, epithelial lining fluid of the lungs, and urinary tract. ${ }^{67}$ The peak plasma concentration of 
tigecycline is in the range of 0.6 to $0.9 \mathrm{mg} / \mathrm{L} .{ }^{67}$ Therefore, it is generally not considered a good option for treatment of patients with bacteremia, severe pneumonia, and urinary tract infection. It did not attain clinical approval for hospitalacquired pneumonia because of a phase 3 study which showed an inferior cure rate compared with imipenem. ${ }^{68}$ This inferiority was primarily driven by those with ventilator-associated pneumonia, which is a relevant clinical presentation for CRE. A higher dose of tigecycline (100 mg every 12 hours) has resulted in a nominally better clinical cure rate compared with imipenem for hospital-acquired pneumonia in a phase 2 study. ${ }^{69}$ This is not an approved dose, and further studies would be required before this approach can be recommended. Overall, despite the in vitro activity of tigecycline against $\mathrm{CPE}$, its use in treatment is usually in the context of combination therapy given the above-mentioned reservations.

\section{Carbapenems}

CPEs exhibit elevated MICs to carbapenems, but the level of resistance is highly variable, ranging from as low as $0.12 \mathrm{mg} / \mathrm{L}$ to $>256 \mathrm{mg} / \mathrm{L}$. For strains with low MICs up to $4 \mathrm{mg} / \mathrm{L}$, prolonged infusion of high-dose carbapenem may achieve sufficient free time above MIC (i.e., $>40 \%$ ) required for bactericidal effect. Limited clinical experience suggests that monotherapy with carbapenem may indeed result in clinical cure when carbapenem MICs of the CPE isolates are low. ${ }^{70}$ However, the majority of CPEs have carbapenem MICs which exceed this range, and there is also a concern for carbapenem therapy leading to elevation of MICs through mutations in porin genes. ${ }^{71}$ Therefore, treatment of CPE infection with carbapenem alone is generally discouraged, perhaps with the exception of rare cases where carbapenem MICs are exceedingly low and the source of infection is well controlled.

A unique approach to treatment of KPC-producing $K$. pneumoniae infection using two carbapenems has been proposed. The rationale is that ertapenem, which has high affinity to the KPC enzyme, would serve as a decoy allowing for the second carbapenem (meropenem or doripenem) to be protected from KPC and bind to the target penicillin binding proteins better. ${ }^{72}$ Anecdotal success of this approach in patients with KPC-producing K. pneumoniae infection has been reported. ${ }^{73}$ However, controlled clinical data would be needed to determine if this is a unique effect from the combination or due to the higher net amount of carbapenems that are administered.

Despite these reservations regarding carbapenem-only regimens, carbapenems appear to constitute an essential element of combination therapy in treating CPE infections, which will be described in more detail later.

\section{Gentamicin}

Aminoglycosides, especially gentamicin, may have a role in the treatment of KPC-producing K. pneumoniae infection. In particular, the global epidemic clone ST258 is more likely to maintain susceptibility to gentamicin compared with other STs. ${ }^{74}$ Gentamicin at a concentration of $10 \mathrm{mg} / \mathrm{L}$ has been shown to exert substantial bactericidal activity against gen- tamicin-susceptible, KPC-producing K. pneumoniae ST258 strains within minutes of exposure. ${ }^{75}$ In a murine thigh infection model, monotherapy with gentamicin was efficacious in the majority of KPC-producing K. pneumoniae strains tested. ${ }^{76}$ In clinical practice, gentamicin is almost always used in the context of combination therapy, often in combination with colistin, a carbapenem, or tigecycline. ${ }^{77}$

In contrast, aminoglycosides are not considered as an option for NDM-producing Enterobacteriaceae because the majority of the isolates produce $16 \mathrm{~S}$ ribosomal RNA methyltransferase, which renders them completely resistant to aminoglycosides. $^{78}$

\section{Fosfomycin}

Fosfomycin is a peptidoglycan synthesis inhibitor that has a wide spectrum of activity ranging from gram-positive to gram-negative bacteria. In the United States, it is available only for oral administration (fosfomycin tromethamine), but an intravenous formulation (fosfomycin disodium) is also available in some European countries. It is a small molecule with negligible protein binding, and is cleared well by glomerular filtration, achieving very high levels in the urine. ${ }^{79}$ The oral formulation is used almost exclusively for treatment of urinary tract infection. The intravenous formulation, on the other hand, has been used for therapy of various types of infections where available. ${ }^{80} \mathrm{CPE}$ isolates including KPCproducing $K$. pneumoniae mostly remain susceptible to fosfomycin and likely could be used for treatment of urinary tract infection. ${ }^{81,82}$ For systemic infections, intravenous fosfomycin is used in combination with another agent (e.g., colistin, tigecycline), making assessment of the efficacy difficult, but it appears to be well tolerated. ${ }^{83}$

\section{Rifampin}

Rifampin is an inhibitor of RNA polymerase and has a very broad spectrum of activity. Its use is limited by rapid emergence of resistance due to amino acid substitutions in the target polymerase; thus, rifampin is always used in combination with other agents. Some studies have reported in vitro or in vivo synergy in the killing of CPE between rifampin and another agent (e.g., tigecycline, colistin) ${ }^{76,84}$ Clinical data are scarce, however, and the significant interactions between rifampin and medications often used in the patient populations prone to CPE infections (e.g., tacrolimus, voriconazole) also limit its potential for use in the treatment of CPE infections.

\section{Doxycycline}

Doxycycline is partially eliminated through glomerular filtration (20-30\%), and achieves adequate concentration in the urine. ${ }^{85}$ Doxycycline and, to a lesser extent, minocycline retain activity against KPC-producing $K$. pneumoniae and have been used successfully in the treatment of urinary tract infection. ${ }^{86,87}$ Limited in vitro data suggest that isolates with a doxycycline MICs close to the susceptibility breakpoint may not be inhibited well by this agent, ${ }^{88}$ and that doxycycline may have some synergy with gentamicin for KPC-producing K. pneumoniae, but synergy with other commonly used drugs 
(e.g., colistin) is not robust. Therefore, its potential use is likely limited to therapy of urinary tract infection in patients who are not systemically ill.

\section{Eravacycline}

Eravacycline is a fluorocycline, with a tetracycline core. Obviously, since it is not a $\beta$-lactam antibiotic, it will not be inhibited by $\beta$-lactamases. In vitro, it has activity against most KPC producers. ${ }^{89}$ It remains to be tested clinically against substantial numbers of patients with infections with CPE.

\section{Ceftazidime-Avibactam}

Avibactam is a new $\beta$-lactamase inhibitor which has activity against many $\beta$-lactamase types, except for those of class $B$ (i.e., metallo- $\beta$-lactamases). Thus, it has useful activity against class A carbapenemases such as the KPC-type. The in vitro effectiveness of the ceftazidime-avibactam combination has been well documented against contemporary isolates, with $\mathrm{MIC}_{50}$ and $\mathrm{MIC}_{90}$ values of 0.5 and $2 \mathrm{mg} / \mathrm{L}$, respectively. ${ }^{90}$ Unfortunately, given the lack of activity of avibactam against metallo- $\beta$-lactamases, ceftazidime-avibactam does not have useful in vitro activity against NDM producers. ${ }^{90}$ At present, there are no published data on the clinical use of ceftazidimeavibactam against CPE infections.

\section{Aztreonam-Avibactam}

The combination of aztreonam and avibactam offers the potential for activity against metallo- $\beta$-lactamase-producing organisms, such as NDM producers. The rationale for this is that aztreonam is stable to the effects of metallo- $\beta$-lactamases. The addition of avibactam will inhibit other produced $\beta$-lactamases allowing aztreonam to act unhindered. The in vitro utility of this approach has been demonstrated against NDM, ${ }^{91}$ IMP, ${ }^{91}$ and VIM producers. ${ }^{92}$ At present, there are no published data on the clinical use of aztreonam-avibactam against CPE infections.

\section{Ceftolozane-Tazobactam}

While ceftolozane-tazobactam has good activity against ESBL-producing strains, it does not appear to have useful activity against $\mathrm{CPE} .^{93}$

\section{Plazomicin}

Plazomicin is a new aminoglycoside which is not affected by most aminoglycoside-modifying enzymes. ${ }^{94}$ Therefore, it has activity against most KPC-producing strains. ${ }^{94}$ Unfortunately, many metallo- $\beta$-lactamase producers also produce $16 \mathrm{~S}$ rRNA methyltransferases rendering plazomicin inactive. ${ }^{95}$ Results of clinical trials on the use of intravenous plazomicin are awaited.

\section{Inhaled Amikacin}

Amikacin has long been available to clinicians in an intravenous formulation. A novel pulmonary drug delivery system is being developed to deliver high concentrations of amikacin to the lung, thereby facilitating treatment of pneumonia caused by multiresistant gram-negative organisms. Results of clinical trials of the use of inhaled amikacin are awaited.

\section{Therapy of CPE Infection: Review of Clinical Evidence}

While data from randomized control studies would best address questions regarding appropriate therapeutic approaches for CPE infections, no such studies have been completed and reported till date. Therefore, information regarding the antimicrobial regimen and clinical outcome of CPE infection are mostly derived from observational studies. Most data have been published for bacteremia caused by KPC-producing K. pneumoniae, which is a frequently encountered condition with high mortality in countries where this organism is endemic (United States, Italy, and Greece). - Table 1 summarizes the findings of 4 such studies that included more than 20 patients. These studies sought to correlate the definitive therapy (or culture-directed therapy; antimicrobial treatment that is given after the culture data including susceptibility testing results become available) started 72 to 96 hours after cultures were collected and the mortality of the patients, either in hospital or at 28 or 30 days. While mortality at 14 days may better reflect the impact from sepsis, patients are usually still on definitive therapy at that point, which is why most studies use 28- or 30-day mortality as the primary outcome variable. The overall mortality is approximately $40 \%$ across these studies.

The available data overall support the superiority of combination therapy (two or more agents active in vitro against the infecting strain) over monotherapy (one agent active in vitro) in terms of patient survival for invasive CPE infections. Here, active agents usually include colistin, tigecycline, and sometimes carbapenems and gentamicin depending on the causative strains. The report by Zarkotou et al was the first to demonstrate this association. ${ }^{96}$ The study was conducted at two hospitals in Greece and included 53 patients with KPC-producing K. pneumoniae bacteremia. Mortality was similar for those who received appropriate or inappropriate empiric therapy. Among 35 of them who survived to receive appropriate definitive therapy (i.e., at least one agent active in vitro), the infection-related mortality was $0 \%$ for 20 patients who received combination therapy and $46.7 \%$ for 15 patients who received monotherapy $(p=0.001$ ). The most common combination was colistin and tigecycline (9 patients; 0\% infection-related mortality), and the most common monotherapy was colistin (7 patients; $66.7 \%$ infection-related mortality). Qureshi et al then examined the outcome of 41 patients at two hospitals in the United States. ${ }^{97}$ Of the 34 patients who received definitive therapy, the 28-day mortality was $13.3 \%$ for 15 patients who received combination therapy and $57.8 \%$ for 19 patients who receive monotherapy $(p=0.01)$. The combinations used were variable, whereas monotherapy largely consisted of colistin (or polymyxin B), tigecycline, or carbapenem. These findings corroborated the results by Zarkotou et al. ${ }^{96}$

Recently, two larger studies on the same topic were presented from Italy and Greece. These studies differed from the aforementioned ones in that colistin and meropenem were generally given at higher doses ( $270 \mathrm{mg}$ a day and $6 \mathrm{~g}$ a day, respectively). A multicenter study conducted in Italy 
Table 1 Observational studies associating therapy and clinical outcome of bacteremia caused by KPC-producing Klebsiella pneumoniae

\begin{tabular}{|c|c|c|c|c|c|c|}
\hline Study & Year & $\begin{array}{l}\text { No. of } \\
\text { patients }\end{array}$ & $\begin{array}{l}\text { Treatment given } \\
\text { (no. of patients) }\end{array}$ & Mortality & Comments & Reference \\
\hline $\begin{array}{l}\text { Zarkotou et al } \\
\text { (Greece) }\end{array}$ & $2008-2010$ & 35 & $\begin{array}{l}\text { Combination } \\
\text { therapy (20) } \\
\text { Monotherapy (15) }\end{array}$ & $\begin{array}{l}0 \% \text { in-hospital }^{\mathrm{b}} \\
46.7 \% \text { in-hospital }^{-}\end{array}$ & $\begin{array}{l}9 \text { of the combination } \\
\text { group received } \\
\text { tigecycline and } \\
\text { colistin }\end{array}$ & 96 \\
\hline $\begin{array}{l}\text { Qureshi et al } \\
\text { (United States) }\end{array}$ & $2005-2009$ & 34 & $\begin{array}{l}\text { Combination } \\
\text { therapy (15) } \\
\text { Monotherapy (19) }\end{array}$ & $\begin{array}{l}13.3 \% \text { at } 28 \mathrm{~d} \\
57.8 \% \text { at } 28 \mathrm{~d}\end{array}$ & $\begin{array}{l}9 \text { patients received } \\
\text { carbapenem- } \\
\text { containing combina- } \\
\text { tions; all were resis- } \\
\text { tant to carbapenems }\end{array}$ & 97 \\
\hline $\begin{array}{l}\text { Tumbarello et al } \\
\text { (Italy) }\end{array}$ & $2010-2011$ & 125 & $\begin{array}{l}\text { Combination } \\
\text { therapy (79) } \\
\text { Tigecycline-colistin } \\
\text { (23) } \\
\text { Tigecycline-genta- } \\
\text { micin (12) } \\
\text { Colistin-gentami- } \\
\text { cin (7) } \\
\text { Tigecycline-colis- } \\
\text { tin-meropenem } \\
(16) \\
\text { Tigecycline-genta- } \\
\text { micin-meropenem } \\
\text { (6) } \\
\text { Monotherapy (46) }\end{array}$ & $\begin{array}{l}34.1 \% \text { at } 30 \mathrm{~d} \\
30.4 \% \\
50.0 \% \\
57.1 \% \\
12.5 \% \\
16.6 \% \\
54.3 \% \text { at } 30 \mathrm{~d}\end{array}$ & $\begin{array}{l}36 \text { patients received } \\
\text { carbapenem-contain- } \\
\text { ing combinations; } \\
\text { mortality was corre- } \\
\text { lated with merope- } \\
\text { nem MICs }\end{array}$ & 98 \\
\hline $\begin{array}{l}\text { Daikos et al } \\
\text { (Greece) }\end{array}$ & $2009-2010$ & $205^{a}$ & $\begin{array}{l}\text { Combination thera- } \\
\text { py (103) } \\
\text { Carbapenem- } \\
\text { containing (31) } \\
\text { Carbapenem- } \\
\text { sparing (72) } \\
\text { Monotherapy (72) }\end{array}$ & $\begin{array}{l}27.2 \% \text { at } 28 d \\
19.3 \% \\
30.6 \% \\
44.4 \% \text { at } 28 d\end{array}$ & $\begin{array}{l}\text { For carbapenem-con- } \\
\text { taining combina- } \\
\text { tions, mortality was } \\
\text { higher for MIC of }>8 \\
\mathrm{mg} / \mathrm{L}(35.5 \%) \text { than } \\
\mathrm{MIC} \text { of } \leq 8 \mathrm{mg} / \mathrm{L} \\
(19.3 \%)\end{array}$ & 99 \\
\hline
\end{tabular}

Abbreviations: KPC, Klebsiella pneumoniae carbapenemase; MICs, minimum inhibitory concentrations.

${ }^{a} 127$ had KPC-producing K. pneumoniae, 36 had KPC/VIM-producing K. pneumoniae, and 42 had VIM-producing K. pneumoniae.

${ }^{\mathrm{b}}$ Mortality attributable to KPC-producing K. pneumoniae bacteremia.

and reported by Tumbarello et al studied 125 patients with bacteremia caused by KPC-producing K. pneumoniae. ${ }^{98}$ The 30-day mortality rates were $34.1 \%$ for 79 patients who received combination therapy and $54.3 \%$ for 46 patients who received monotherapy $(p=0.02)$. Notably, the lowest mortality rates within the combination group were observed for those who received three active agents (12.5\% for 16 patients treated with tigecycline, colistin, and meropenem and $16.6 \%$ for 6 patients who received tigecycline, gentamicin, and meropenem), and lower meropenem MICs were associated with lower mortality in the combination therapy group. Finally, Daikos et al examined the outcome of 205 patients with bacteremia caused by carbapenemase-producing $K$. pneumoniae, 163 of which produced KPC, at two hospitals in Greece. ${ }^{99}$ The rate of resistance to colistin was high at $25.4 \%$. Of the 175 patients who received active definitive therapy, 103 received combination therapy and 72 received monotherapy. The 28-day mortality rates were 27.2 and $44.4 \%$, respectively ( $p=0.003$ ), and the beneficial effect of combination therapy was maximized among those with rapidly fatal underlying diseases or septic shock. In addition, the mortality rate was seen among patients given carbape- nem-containing combinations (19.3\%), and lower carbapenem MICs were associated with lower mortality as was observed in the study by Tumbarello et al.

A recent systematic review by Tzouvelekis et al has compiled reports on the clinical outcome of CPE infections (mostly KPC producers with some VIM and OXA-48 producers) in 889 patients, which included those in the aforementioned studies. ${ }^{100}$ Among them, 441 received combination therapy, 346 received monotherapy, and 102 received inappropriate therapy (i.e., no active agent in vitro). The mortality rates of monotherapy were $40.1 \%$ for carbapenem, $41.1 \%$ for tigecycline, and $42.8 \%$ for colistin, whereas the mortality for inappropriate therapy was $46.1 \%$. In contrast, the mortality rates for combination therapy were $30.7 \%$ for carbapenem-sparing combinations and $18.8 \%$ for carbapenem-containing combinations. Again, these data are all derived from observational, uncontrolled studies. However, the two arms (combination therapy and monotherapy) are comparable in terms of demographics, underlying diseases, and severity of illness across these studies. In addition, the bias, if any, would be in the direction of adopting combination therapy for more ill-appearing patients for whom higher 
mortality would be anticipated. Therefore, we recommend for the time being that invasive infections caused by CPE be treated with two in vitro active agents that include a carbapenem, with the second agent selected from colistin, tigecycline, or gentamicin depending on susceptibility. It remains an open question whether the addition of a third agent is warranted when the carbapenem MIC is high, as the benefits of combination therapy should be weighed against potential harms in terms of costs, adverse events, and interference with other therapy through the intravenous access, among others. Randomized clinical trials aimed at comparing colistin alone and colistin plus meropenem for CPE infections are ongoing in the United States and European Union (NCT01597973 and NCT01732250). The findings from these studies, when available, are expected to provide valuable insights on the efficacy of these approaches when pharmacokinetically optimized doses are used for these agents.

Currently, there are no substantial data correlating treatment and clinical outcome for infections caused by NDMproducing Enterobacteriaceae.

\section{Summary}

CPE is one of the biggest infectious disease threats that have emerged in hospitals worldwide in the last decade. CPE infections are difficult to manage because of limited treatment options and are associated with high mortality. KPC-producing K. pneumoniae is the most prevalent CPE and the best studied as well. Treatment of invasive infections such as bacteremia usually consists of two active agents depending on the susceptibility patterns of the infecting strain (e.g., colistin and tigecycline, colistin and meropenem, meropenem and tigecycline, and meropenem and gentamicin), as this approach has been associated with lower patient mortality compared with treatment with a single active agent in multiple observational studies. At the same time, dosing for each agent should be optimized by using high doses, and in the case of colistin and tigecycline, with a loading dose. On the other hand, uncomplicated urinary tract infections caused by CPE can be safely managed with a variety of single agents with good clinical outcome. Of concern is the increasing number of reports documenting CPE resistance to colistin and tigecycline as we use more of these "salvage" agents for therapy. How these pandrug-resistant cases can be best managed remains an open question.

\section{Acknowledgment}

Y.D. acknowledges support from the National Institutes of Health through research grants R21AI107302, R01AI104895.

\section{References}

1 Rasmussen BA, Bush K. Carbapenem-hydrolyzing $\beta$-lactamases. Antimicrob Agents Chemother 1997;41(2):223-232

2 Martínez-Martínez L, Pascual A, Hernández-Allés S, et al. Roles of $\beta$-lactamases and porins in activities of carbapenems and cephalosporins against Klebsiella pneumoniae. Antimicrob Agents Chemother 1999;43(7):1669-1673
3 Yigit $\mathrm{H}$, Queenan AM, Anderson GJ, et al. Novel carbapenemhydrolyzing $\beta$-lactamase, KPC-1, from a carbapenem-resistant strain of Klebsiella pneumoniae. Antimicrob Agents Chemother 2001;45(4):1151-1161

4 Bradford PA, Bratu S, Urban C, et al. Emergence of carbapenemresistant Klebsiella species possessing the class A carbapenemhydrolyzing KPC-2 and inhibitor-resistant TEM-30 $\beta$-lactamases in New York City. Clin Infect Dis 2004;39(1):55-60

5 Bratu S, Landman D, Haag R, et al. Rapid spread of carbapenemresistant Klebsiella pneumoniae in New York City: a new threat to our antibiotic armamentarium. Arch Intern Med 2005;165(12): 1430-1435

6 Kitchel B, Rasheed JK, Patel JB, et al. Molecular epidemiology of KPC-producing Klebsiella pneumoniae isolates in the United States: clonal expansion of multilocus sequence type 258. Antimicrob Agents Chemother 2009;53(8):3365-3370

7 Gupta N, Limbago BM, Patel JB, Kallen AJ. Carbapenem-resistant Enterobacteriaceae: epidemiology and prevention. Clin Infect Dis 2011;53(1):60-67

8 Samra Z, Ofir O, Lishtzinsky Y, Madar-Shapiro L, Bishara J. Outbreak of carbapenem-resistant Klebsiella pneumoniae producing KPC-3 in a tertiary medical centre in Israel. Int J Antimicrob Agents 2007;30(6):525-529

9 Navon-Venezia S, Leavitt A, Schwaber MJ, et al; Israeli KPC Kpn Study Group. First report on a hyperepidemic clone of KPC-3producing Klebsiella pneumoniae in Israel genetically related to a strain causing outbreaks in the United States. Antimicrob Agents Chemother 2009;53(2):818-820

10 Schwaber MJ, Lev B, Israeli A, et al; Israel Carbapenem-Resistant Enterobacteriaceae Working Group. Containment of a countrywide outbreak of carbapenem-resistant Klebsiella pneumoniae in Israeli hospitals via a nationally implemented intervention. Clin Infect Dis 2011;52(7):848-855

11 Schwaber MJ, Carmeli Y. An ongoing national intervention to contain the spread of carbapenem-resistant enterobacteriaceae. Clin Infect Dis 2014;58(5):697-703

12 Maltezou HC, Giakkoupi P, Maragos A, et al. Outbreak of infections due to KPC-2-producing Klebsiella pneumoniae in a hospital in Crete (Greece). J Infect 2009;58(3):213-219

13 Psichogiou M, Tassios PT, Avlamis A, et al. Ongoing epidemic of

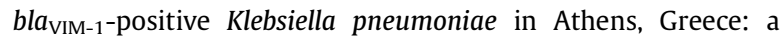
prospective survey. J Antimicrob Chemother 2008;61(1):59-63

14 Agodi A, Voulgari E, Barchitta M, et al. Containment of an outbreak of KPC-3-producing Klebsiella pneumoniae in Italy. J Clin Microbiol 2011;49(11):3986-3989

15 Giani T, Pini B, Arena F, et al. Epidemic diffusion of KPC carbapenemase-producing Klebsiella pneumoniae in Italy: results of the first countrywide survey, 15 May to 30 June 2011. Euro Surveill 2013;18(22)

16 Munoz-Price LS, Poirel L, Bonomo RA, et al. Clinical epidemiology of the global expansion of Klebsiella pneumoniae carbapenemases. Lancet Infect Dis 2013;13(9):785-796

17 Woodford N, Turton JF, Livermore DM. Multiresistant Gramnegative bacteria: the role of high-risk clones in the dissemination of antibiotic resistance. FEMS Microbiol Rev 2011;35(5): 736-755

18 Warburg G, Hidalgo-Grass C, Partridge SR, et al. A carbapenemresistant Klebsiella pneumoniae epidemic clone in Jerusalem: sequence type 512 carrying a plasmid encoding $a a c\left(6^{\prime}\right)-I b$. J Antimicrob Chemother 2012;67(4):898-901

19 Capone A, Giannella M, Fortini D, et al; SEERBIO-GRAB network. High rate of colistin resistance among patients with carbapenemresistant Klebsiella pneumoniae infection accounts for an excess of mortality. Clin Microbiol Infect 2013;19(1):E23-E30

20 Qi Y, Wei Z, Ji S, Du X, Shen P, Yu Y. ST11, the dominant clone of KPC-producing Klebsiella pneumoniae in China. J Antimicrob Chemother 2011;66(2):307-312 
21 Pereira PS, de Araujo CF, Seki LM, Zahner V, Carvalho-Assef AP, Asensi MD. Update of the molecular epidemiology of KPC-2producing Klebsiella pneumoniae in Brazil: spread of clonal complex 11 (ST11, ST437 and ST340). J Antimicrob Chemother 2013;68(2):312-316

22 Ruiz-Garbajosa P, Curiao T, Tato M, et al. Multiclonal dispersal of KPC genes following the emergence of non-ST258 KPC-producing Klebsiella pneumoniae clones in Madrid, Spain. J Antimicrob Chemother 2013;68(11):2487-2492

23 O'Hara JA, Hu F, Ahn C, et al. Molecular epidemiology of KPCproducing Escherichia coli: occurrence of ST131-fimH30 subclone harboring pKpQIL-like IncFIIk plasmid. Antimicrob Agents Chemother 2014;58(7):4234-4237

24 Ahn C, Syed A, Hu F, O'Hara JA, Rivera JI, Doi Y. Microbiological features of KPC-producing Enterobacter isolates identified in a U. S. hospital system. Diagn Microbiol Infect Dis 2014;80(2): 154-158

25 Robledo IE, Aquino EE, Vázquez GJ. Detection of the KPC gene in Escherichia coli, Klebsiella pneumoniae, Pseudomonas aeruginosa, and Acinetobacter baumannii during a PCR-based nosocomial surveillance study in Puerto Rico. Antimicrob Agents Chemother 2011;55(6):2968-2970

26 Yong D, Toleman MA, Giske CG, et al. Characterization of a new metallo- $\beta$-lactamase gene, $b l a_{\mathrm{NDM}-1}$, and a novel erythromycin esterase gene carried on a unique genetic structure in Klebsiella pneumoniae sequence type 14 from India. Antimicrob Agents Chemother 2009;53(12):5046-5054

27 Castanheira M, Deshpande LM, Mathai D, Bell JM, Jones RN, Mendes RE. Early dissemination of NDM-1- and OXA-181-producing Enterobacteriaceae in Indian hospitals: report from the SENTRY Antimicrobial Surveillance Program, 2006-2007. Antimicrob Agents Chemother 2011;55(3):1274-1278

28 Castanheira M, Deshpande LM, Farrell SE, Shetye S, Shah N, Jones RN. Update on the prevalence and genetic characterization of NDM-1-producing Enterobacteriaceae in Indian hospitals during 2010. Diagn Microbiol Infect Dis 2013;75(2):210-213

29 Day KM, Ali S, Mirza IA, et al. Prevalence and molecular characterization of Enterobacteriaceae producing NDM-1 carbapenemase at a military hospital in Pakistan and evaluation of two chromogenic media. Diagn Microbiol Infect Dis 2013;75(2): 187-191

30 Zowawi HM, Balkhy HH, Walsh TR, Paterson DL. $\beta$-Lactamase production in key gram-negative pathogen isolates from the Arabian Peninsula. Clin Microbiol Rev 2013;26(3):361-380

31 Meletis G, Oustas E, Bagkeri M. Carbapenemase reports from the Balkans: a systematic review. Infez Med 2014;22(2):85-106

32 Walsh TR, Weeks J, Livermore DM, Toleman MA. Dissemination of NDM-1 positive bacteria in the New Delhi environment and its implications for human health: an environmental point prevalence study. Lancet Infect Dis 2011;11(5):355-362

33 Savard P, Gopinath R, Zhu W, et al. First NDM-positive Salmonella sp. strain identified in the United States. Antimicrob Agents Chemother 2011;55(12):5957-5958

34 Darley E, Weeks J, Jones L, et al. NDM-1 polymicrobial infections including Vibrio cholerae. Lancet 2012;380(9850):1358

35 Poirel L, Héritier C, Tolün V, Nordmann P. Emergence of oxacillinase-mediated resistance to imipenem in Klebsiella pneumoniae. Antimicrob Agents Chemother 2004;48(1):15-22

36 Poirel L, Potron A, Nordmann P. OXA-48-like carbapenemases: the phantom menace. J Antimicrob Chemother 2012;67(7): 1597-1606

37 Mathers AJ, Hazen KC, Carroll J, et al. First clinical cases of OXA48-producing carbapenem-resistant Klebsiella pneumoniae in the United States: the "menace" arrives in the new world. J Clin Microbiol 2013;51(2):680-683

38 Thaden JT, Lewis SS, Hazen KC, et al. Rising rates of carbapenemresistant enterobacteriaceae in community hospitals: a mixedmethods review of epidemiology and microbiology practices in a network of community hospitals in the southeastern United States. Infect Control Hosp Epidemiol 2014;35(8):978-983

39 Nordmann P, Gniadkowski M, Giske CG, Poirel L, Woodford N, Miriagou V; European Network on Carbapenemases. Identification and screening of carbapenemase-producing Enterobacteriaceae. Clin Microbiol Infect 2012;18(5):432-438

40 Anderson KF, Lonsway DR, Rasheed JK, et al. Evaluation of methods to identify the Klebsiella pneumoniae carbapenemase in Enterobacteriaceae. J Clin Microbiol 2007;45(8):2723-2725

41 Nordmann P, Poirel L, Dortet L. Rapid detection of carbapenemase-producing Enterobacteriaceae. Emerg Infect Dis 2012; 18(9):1503-1507

42 Vasoo S, Cunningham SA, Kohner PC, et al. Comparison of a novel, rapid chromogenic biochemical assay, the Carba NP test, with the modified Hodge test for detection of carbapenemase-producing Gram-negative bacilli. J Clin Microbiol 2013;51(9):3097-3101

43 Doi Y, Potoski BA, Adams-Haduch JM, Sidjabat HE, Pasculle AW, Paterson DL. Simple disk-based method for detection of Klebsiella pneumoniae carbapenemase-type $\beta$-lactamase by use of a boronic acid compound. J Clin Microbiol 2008;46(12):4083-4086

44 Tsakris A, Themeli-Digalaki K, Poulou A, et al. Comparative evaluation of combined-disk tests using different boronic acid compounds for detection of Klebsiella pneumoniae carbapenemase-producing enterobacteriaceae clinical isolates. J Clin Microbiol 2011;49(8):2804-2809

45 Hattori T, Kawamura K, Arakawa Y. Comparison of test methods for detecting metallo- $\beta$-lactamase-producing Gram-negative bacteria. Jpn J Infect Dis 2013;66(6):512-518

46 Cuzon G, Naas T, Bogaerts P, Glupczynski Y, Nordmann P. Evaluation of a DNA microarray for the rapid detection of extendedspectrum $\beta$-lactamases (TEM, SHV and CTX-M), plasmid-mediated cephalosporinases (CMY-2-like, DHA, FOX, ACC-1, ACT/MIR and CMY-1-like/MOX) and carbapenemases (KPC, OXA-48, VIM, IMP and NDM). J Antimicrob Chemother 2012;67(8):1865-1869

47 Lolans K, Calvert K, Won S, Clark J, Hayden MK. Direct ertapenem disk screening method for identification of KPC-producing Klebsiella pneumoniae and Escherichia coli in surveillance swab specimens. J Clin Microbiol 2010;48(3):836-841

48 Adler A, Navon-Venezia S, Moran-Gilad J, Marcos E, Schwartz D, Carmeli Y. Laboratory and clinical evaluation of screening agar plates for detection of carbapenem-resistant Enterobacteriaceae from surveillance rectal swabs. J Clin Microbiol 2011;49(6): 2239-2242

49 Landman D, Salvani JK, Bratu S, Quale J. Evaluation of techniques for detection of carbapenem-resistant Klebsiella pneumoniae in stool surveillance cultures. J Clin Microbiol 2005;43(11):5639-5641

50 Vasoo S, Cunningham SA, Kohner PC, et al. Rapid and direct realtime detection of $b l a_{\mathrm{KPC}}$ and $b l a_{\mathrm{NDM}}$ from surveillance samples. J Clin Microbiol 2013;51(11):3609-3615

51 Bergen PJ, Landersdorfer CB, Zhang J, et al. Pharmacokinetics and pharmacodynamics of 'old' polymyxins: what is new? Diagn Microbiol Infect Dis 2012;74(3):213-223

52 Akajagbor DS, Wilson SL, Shere-Wolfe KD, Dakum P, Charurat ME, Gilliam BL. Higher incidence of acute kidney injury with intravenous colistimethate sodium compared with polymyxin B in critically ill patients at a tertiary care medical center. Clin Infect Dis 2013;57(9):1300-1303

53 Garonzik SM, Li J, Thamlikitkul V, et al. Population pharmacokinetics of colistin methanesulfonate and formed colistin in critically ill patients from a multicenter study provide dosing suggestions for various categories of patients. Antimicrob Agents Chemother 2011;55(7):3284-3294

54 Florescu DF, Qiu F, McCartan MA, Mindru C, Fey PD, Kalil AC. What is the efficacy and safety of colistin for the treatment of ventilatorassociated pneumonia? A systematic review and meta-regression. Clin Infect Dis 2012;54(5):670-680

55 Mohamed AF, Karaiskos I, Plachouras D, et al. Application of a loading dose of colistin methanesulfonate in critically ill patients: 
population pharmacokinetics, protein binding, and prediction of bacterial kill. Antimicrob Agents Chemother 2012;56(8): 4241-4249

56 Li J, Milne RW, Nation RL, Turnidge JD, Smeaton TC, Coulthard K. Pharmacokinetics of colistin methanesulphonate and colistin in rats following an intravenous dose of colistin methanesulphonate. J Antimicrob Chemother 2004;53(5):837-840

57 Plachouras D, Karvanen M, Friberg LE, et al. Population pharmacokinetic analysis of colistin methanesulfonate and colistin after intravenous administration in critically ill patients with infections caused by gram-negative bacteria. Antimicrob Agents Chemother 2009;53(8):3430-3436

58 Imberti R, Cusato M, Villani P, et al. Steady-state pharmacokinetics and BAL concentration of colistin in critically Ill patients after IV colistin methanesulfonate administration. Chest 2010;138(6): 1333-1339

59 Kwa AL, Lim TP, Low JG, et al. Pharmacokinetics of polymyxin B1 in patients with multidrug-resistant Gram-negative bacterial infections. Diagn Microbiol Infect Dis 2008;60(2):163-167

60 Mammina C, Bonura C, Di Bernardo F, et al. Ongoing spread of colistin-resistant Klebsiella pneumoniae in different wards of an acute general hospital, Italy, June to December 2011. Euro Surveill 2012;17(33):20248

61 Bogdanovich T, Adams-Haduch JM, Tian GB, et al. Colistin-resistant, Klebsiella pneumoniae carbapenemase (KPC)-producing Klebsiella pneumoniae belonging to the international epidemic clone ST258. Clin Infect Dis 2011;53(4):373-376

62 Mezzatesta ML, Gona F, Caio C, et al. Outbreak of KPC-3-producing, and colistin-resistant, Klebsiella pneumoniae infections in two Sicilian hospitals. Clin Microbiol Infect 2011;17(9): 1444-1447

63 Al-Agamy MH, Shibl AM, Elkhizzi NA, Meunier D, Turton JF, Livermore DM. Persistence of Klebsiella pneumoniae clones with OXA48 or NDM carbapenemases causing bacteraemias in a Riyadh hospital. Diagn Microbiol Infect Dis 2013;76(2):214-216

64 Arpin C, Noury P, Boraud D, et al. NDM-1-producing Klebsiella pneumoniae resistant to colistin in a French community patient without history of foreign travel. Antimicrob Agents Chemother 2012;56(6):3432-3434

65 van Duin D, Perez F, Rudin SD, et al. Surveillance of carbapenemresistant Klebsiella pneumoniae: tracking molecular epidemiology and outcomes through a regional network. Antimicrob Agents Chemother 2014;58(7):4035-4041

66 van Duin D, Cober ED, Richter SS, et al. Tigecycline therapy for carbapenem-resistant Klebsiella pneumoniae (CRKP) bacteriuria leads to tigecycline resistance. Clin Microbiol Infect 2014;20(12): 01117-01120

67 Barbour A, Schmidt S, Ma B, et al. Clinical pharmacokinetics and pharmacodynamics of tigecycline. Clin Pharmacokinet 2009; 48(9):575-584

68 Freire AT, Melnyk V, Kim MJ, et al; 311 Study Group. Comparison of tigecycline with imipenem/cilastatin for the treatment of hospital-acquired pneumonia. Diagn Microbiol Infect Dis 2010; 68(2):140-151

69 Ramirez J, Dartois N, Gandjini H, Yan JL, Korth-Bradley J, McGovern PC. Randomized phase 2 trial to evaluate the clinical efficacy of two high-dosage tigecycline regimens versus imipenem-cilastatin for treatment of hospital-acquired pneumonia. Antimicrob Agents Chemother 2013;57(4):1756-1762

70 Tzouvelekis LS, Markogiannakis A, Psichogiou M, Tassios PT, Daikos GL. Carbapenemases in Klebsiella pneumoniae and other Enterobacteriaceae: an evolving crisis of global dimensions. Clin Microbiol Rev 2012;25(4):682-707

71 Clancy CJ, Chen L, Hong JH, et al. Mutations of the ompK36 porin gene and promoter impact responses of sequence type 258, KPC2-producing Klebsiella pneumoniae strains to doripenem and doripenem-colistin. Antimicrob Agents Chemother 2013; 57(11):5258-5265
72 Bulik CC, Nicolau DP. Double-carbapenem therapy for carbapenemase-producing Klebsiella pneumoniae. Antimicrob Agents Chemother 2011;55(6):3002-3004

73 Giamarellou H, Galani L, Baziaka F, Karaiskos I. Effectiveness of a double-carbapenem regimen for infections in humans due to carbapenemase-producing pandrug-resistant Klebsiella pneumoniae. Antimicrob Agents Chemother 2013;57(5):2388-2390

74 Naparstek L, Carmeli Y, Navon-Venezia S, Banin E. Biofilm formation and susceptibility to gentamicin and colistin of extremely drug-resistant KPC-producing Klebsiella pneumoniae. J Antimicrob Chemother 2014;69(4):1027-1034

75 Clancy CJ, Hao B, Shields RK, et al. Doripenem, gentamicin, and colistin, alone and in combinations, against gentamicin-susceptible, KPC-producing Klebsiella pneumoniae strains with various ompK36 genotypes. Antimicrob Agents Chemother 2014;58(6): 3521-3525

76 Michail G, Labrou M, Pitiriga V, et al. Activity of tigecycline in combination with colistin, meropenem, rifampin, or gentamicin against KPC-producing Enterobacteriaceae in a murine thigh infection model. Antimicrob Agents Chemother 2013;57(12): 6028-6033

77 Kontopidou F, Giamarellou H, Katerelos P, et al; Group for the Study of KPC-producing Klebsiella pneumoniae infections in intensive care units. Infections caused by carbapenem-resistant Klebsiella pneumoniae among patients in intensive care units in Greece: a multi-centre study on clinical outcome and therapeutic options. Clin Microbiol Infect 2014;20(2):0117-0123

78 Livermore DM, Mushtaq S, Warner M, et al. Activity of aminoglycosides, including ACHN-490, against carbapenem-resistant Enterobacteriaceae isolates. J Antimicrob Chemother 2011;66(1):48-53

79 Parker S, Lipman J, Koulenti D, Dimopoulos G, Roberts JA. What is the relevance of fosfomycin pharmacokinetics in the treatment of serious infections in critically ill patients? A systematic review. Int J Antimicrob Agents 2013;42(4):289-293

80 Dinh A, Salomon J, Bru JP, Bernard L. Fosfomycin: efficacy against infections caused by multidrug-resistant bacteria. Scand J Infect Dis 2012;44(3):182-189

81 Endimiani A, Patel G, Hujer KM, et al. In vitro activity of fosfomycin against bla $_{\mathrm{KP}}$-containing Klebsiella pneumoniae isolates, including those nonsusceptible to tigecycline and/or colistin. Antimicrob Agents Chemother 2010;54(1):526-529

82 Livermore DM, Warner M, Mushtaq S, Doumith M, Zhang J, Woodford N. What remains against carbapenem-resistant Enterobacteriaceae? Evaluation of chloramphenicol, ciprofloxacin, colistin, fosfomycin, minocycline, nitrofurantoin, temocillin and tigecycline. Int J Antimicrob Agents 2011;37(5):415-419

83 Pontikis K, Karaiskos I, Bastani S, et al. Outcomes of critically ill intensive care unit patients treated with fosfomycin for infections due to pandrug-resistant and extensively drug-resistant carbapenemase-producing Gram-negative bacteria. Int J Antimicrob Agents 2014;43(1):52-59

84 Tängdén T, Hickman RA, Forsberg P, Lagerbäck P, Giske CG, Cars O. Evaluation of double- and triple-antibiotic combinations for VIMand NDM-producing Klebsiella pneumoniae by in vitro time-kill experiments. Antimicrob Agents Chemother 2014;58(3): 1757-1762

85 Whelton A, Nightingale SD, Carter GG, Gordon LS, Bryant HH, Walker WG. Pharmacokinetic characteristics of doxycycline accumulation in normal and severely diseased kidneys. J Infect Dis 1975;132(4):467-471

86 Qureshi ZA, Syed A, Clarke LG, Doi Y, Shields RK. Epidemiology and clinical outcomes of patients with carbapenem-resistant Klebsiella pneumoniae bacteriuria. Antimicrob Agents Chemother 2014;58(6):3100-3104

87 Alexander BT, Marschall J, Tibbetts RJ, Neuner EA, Dunne WM Jr, Ritchie DJ. Treatment and clinical outcomes of urinary tract infections caused by KPC-producing Enterobacteriaceae in a retrospective cohort. Clin Ther 2012;34(6):1314-1323 
88 Bratu S, Tolaney P, Karumudi U, et al. Carbapenemase-producing Klebsiella pneumoniae in Brooklyn, NY: molecular epidemiology and in vitro activity of polymyxin $B$ and other agents. J Antimicrob Chemother 2005;56(1):128-132

89 Abdallah M, Olafisoye O, Cortes C, Urban C, Landman D, Quale J. Activity of eravacycline against Enterobacteriaceae and Acinetobacter baumannii, including multidrug-resistant isolates, from New York City. Antimicrob Agents Chemother 2014 [Epub ahead of print]

90 Castanheira M, Farrell SE, Krause KM, Jones RN, Sader HS. Contemporary diversity of $\beta$-lactamases among Enterobacteriaceae in the nine U.S. census regions and ceftazidime-avibactam activity tested against isolates producing the most prevalent $\beta$-lactamase groups. Antimicrob Agents Chemother 2014;58(2): 833-838

91 Wang X, Zhang F, Zhao C, et al. In vitro activities of ceftazidimeavibactam and aztreonam-avibactam against 372 Gram-negative bacilli collected in 2011 and 2012 from 11 teaching hospitals in China. Antimicrob Agents Chemother 2014;58(3):1774-1778

92 Li H, Estabrook M, Jacoby GA, Nichols WW, Testa RT, Bush K. In vitro susceptibility of characterized $\beta$-lactamase-producing strains tested with avibactam combinations. Antimicrob Agents Chemother 2014 [Epub ahead of print]

93 Sader HS, Farrell DJ, Castanheira M, Flamm RK, Jones RN. Antimicrobial activity of ceftolozane/tazobactam tested against Pseudomonas aeruginosa and Enterobacteriaceae with various resistance patterns isolated in European hospitals (2011-12). J Antimicrob Chemother 2014;69(10):2713-2722
94 Almaghrabi R, Clancy CJ, Doi Y, et al. Carbapenem-resistant Klebsiella pneumoniae strains exhibit diversity in aminoglycoside-modifying enzymes, which exert differing effects on plazomicin and other agents. Antimicrob Agents Chemother 2014; 58(8):4443-4451

95 O'Hara JA, McGann P, Snesrud EC, et al. Novel 16S rRNA methyltransferase RmtH produced by Klebsiella pneumoniae associated with war-related trauma. Antimicrob Agents Chemother 2013; 57(5):2413-2416

96 Zarkotou O, Pournaras S, Tselioti P, et al. Predictors of mortality in patients with bloodstream infections caused by KPC-producing Klebsiella pneumoniae and impact of appropriate antimicrobial treatment. Clin Microbiol Infect 2011;17(12):1798-1803

97 Qureshi ZA, Paterson DL, Potoski BA, et al. Treatment outcome of bacteremia due to KPC-producing Klebsiella pneumoniae: superiority of combination antimicrobial regimens. Antimicrob Agents Chemother 2012;56(4):2108-2113

98 Tumbarello M, Viale P, Viscoli C, et al. Predictors of mortality in bloodstream infections caused by Klebsiella pneumoniae carbapenemase-producing K. pneumoniae: importance of combination therapy. Clin Infect Dis 2012;55(7):943-950

99 Daikos GL, Tsaousi S, Tzouvelekis LS, et al. Carbapenemase-producing Klebsiella pneumoniae bloodstream infections: lowering mortality by antibiotic combination schemes and the role of carbapenems. Antimicrob Agents Chemother 2014;58(4):2322-2328

100 Tzouvelekis LS, Markogiannakis A, Piperaki E, Souli M, Daikos GL. Treating infections caused by carbapenemase-producing Enterobacteriaceae. Clin Microbiol Infect 2014;20(9):862-872 